\title{
The $\tau$ decay library TAUOLA, version 2.4
}

\author{
S. Jadach and Z. Wa̧s \\ TH DIVISION \\ CERN, 1211 Geneva, Switzerland \\ and \\ Institute of Nuclear Physics, Kraków, ul. Kawiory 26a, Poland \\ and \\ R. Decker and J. H. Kühn \\ Institut für Theoretische Teilchenphysik, Universität Karlsruhe, \\ W-7500 Karlsruhe, Germany
}

\begin{abstract}
The $\tau$ decay library TAUOLA has been enriched. The present version of the program includes more than twenty decay channels, including: (a) leptonic modes $\tau^{-} \rightarrow$ $\bar{\nu} \nu e^{-}(\gamma), \bar{\nu} \nu \mu^{-}(\gamma)$, (b) one-meson modes: $\nu \pi^{-}, \nu K^{-},(\mathrm{c})$ two-meson modes: $\nu \pi^{-} \pi^{0}\left(\rho^{-}\right)$, $\nu K^{-} \pi^{0}\left(K^{*-}\right),(\mathrm{d})$ three-meson modes: $\nu \pi^{-} 2 \pi^{0}\left(a_{1}^{-}\right), \nu 2 \pi^{-} \pi^{+}\left(a_{1}^{-}\right), \nu K^{+} K^{-} \pi^{-}, \nu \bar{K}^{0} K^{0} \pi^{-}$, $\nu K^{-} \bar{K}^{0} \pi^{0}, \nu K^{-} \pi^{0} \pi^{0}, \nu K^{-} \pi^{-} \pi^{+}, \nu K^{0} \pi^{0} \pi^{-}, \nu \eta \pi^{-} \pi^{0}$, (e) four-pion modes: $\nu \pi^{-} 3 \pi^{0}$, $\nu 2 \pi^{-} \pi^{+} \pi^{0}$ (including the $\omega \pi^{-}$contribution), (f) multi-pion modes: $\nu n \pi^{ \pm, 0}$ with $n>4$, and finally the decay $\tau^{-} \rightarrow \nu \pi^{-} \pi^{0} \gamma$ from the $\omega \rightarrow \pi \gamma$.

Complete $\mathrm{O}(\alpha)$ QED corrections are included in the leptonic decay channels, and for other decay channels an interface is provided to the PHOTOS Monte Carlo generator for approximate simulation of the QED corrections. An interface to the $\tau$ pair production generators KORALB and KORALZ is also included. Final-state M.C. events are coded in the common block proposed by the Particle Data Group.
\end{abstract}

CERN-TH. 6793/93

February, 1993 


\section{NEW VERSION SUMMARY}

Title of the program: TAUOLA, version: 2.4

Reference to original program: Comp. Phys. Comm. 64 (1991) 275.

Authors of original program: S. Jadach J. H. Kühn and Z. Wạs

Computer: IBM 3090; Installation: CERN

Operating system: VM/CMS

Programing language used: FORTRAN 77

High speed storage required: 45000 words

No. of bits in a word: 32

Peripherals used: Line printer

No. of cards in combined program and test deck: 8000

Keywords: radiative corrections, heavy lepton $\tau$, Monte Carlo simulation, quantum electrodynamics, spin polarization, electro-weak effects

Comparison with the previous version, and reasons for the new version: The $\tau$ decay library [2] is enriched by new decay modes involving kaons: $\tau^{-} \rightarrow \nu K^{+} K^{-} \pi^{-}, \nu \bar{K}^{0} K^{0} \pi^{-}$, $\nu K^{-} K^{0} \pi^{0}, \nu K^{-} \pi^{0} \pi^{0}, \nu K^{-} \pi^{-} \pi^{+}, \nu K^{0} \pi^{0} \pi^{-}, \nu \eta \pi^{-} \pi^{0}$, the $\omega \pi$ contribution to $\nu \pi^{-} \pi^{0} \gamma$. For the $\tau$ decay modes into four pions $\tau^{-} \rightarrow \nu 2 \pi^{-} \pi^{+} \pi^{0}, \pi^{-} 3 \pi^{0}$, the simplified dynamical treatment has been replaced by a more appropriate one, in particular, the $\omega \pi$ contribution and $\rho$ enhancement in $2 \pi^{-} \pi^{+} \pi^{0}$ channel have been implemented.

Typical running time: 37 events per (IBM 3090168 units) sec., in case of all decay modes populated uniformly.

Limitations of the solution: For decays into five and six pions a simplified dynamical treatment has been adopted.

References:

1. S. Jadach, J. H. Kühn, and Z. Wạs, Comp. Phys. Comm. 64 (1991) 275.

2. S. Jadach, M. Jeżabek, J. H. Kühn, and Z. Wąs, Comp. Phys. Comm. 70 (1992) 69.

\section{INTRODUCTION}

Since the publication of the first version of the TAUOLA library [1] substantial progress in the experimental measurement of the $\tau$ lepton has been achieved. This stimulated us to improve our $\tau$ decay Monte Carlo program. There are four main directions of improvements: 
1. Inclusion of new decay modes,

2. More precise treatment of the matrix element,

3. More flexible treatment of the steering parameters, and more efficient generation,

4. More accurate determination of the physical precision of the generator.

In the present update we improve on the first three points, and the improvement on the physical precision is still not satisfactory. This is to be contrasted with many QED Monte Carlo programs where the precision both technical and physical is treated very carefully (see e.g. [2]). In fact we hardly at all address the question of the physical precision of our program. For hadronic decays this is to some extent understandable, as no rigorous theory of low-energy hadronic phenomena exists. In fact, the shapes of many form factors used in the program are directly or indirectly related to previously measured hadronic cross sections and distributions.

The situation with the technical precision is better. Some distributions are obtained using non-Monte-Carlo techniques employing the same assumptions as in the Monte Carlo program. Such a comparison thus checks the technical accuracy of the two methods. The analytical tests were, however, often performed with downgraded (with respect to MC), numerically easy to integrate matrix elements.

Let us now present the improvements of the present version of TAUOLA.

1. We introduce the following eight new $\tau$ decay modes: $\tau^{-} \rightarrow \nu K^{+} K^{-} \pi^{-}, \nu \bar{K}^{0} K^{0} \pi^{+}$, $\nu K^{-} K^{0} \pi^{0}, \nu K^{-} \pi^{0} \pi^{0}, \nu K^{-} \pi^{-} \pi^{+}, \nu K^{0} \pi^{0} \pi^{-}, \nu \eta \pi^{0} \pi^{-}$, and the $\omega \pi$ contribution to $\nu \pi^{-} \pi^{0} \gamma$

2. We improve matrix elements and generation algorithm for the following two decay modes: $\tau^{-} \rightarrow \nu 2 \pi^{-} \pi^{+} \pi^{0}, \nu \pi^{-} 3 \pi^{0}$,

3. We provide an option to add to the dominant $a_{1}$ contribution a hypothetical scalar resonance $\left(\pi^{\prime}\right)$ in the $\tau^{-} \rightarrow \nu \pi^{-} 2 \pi^{0}$ and $\nu 2 \pi^{-} \pi^{+}$decay channels.

4. The general organization of the program is left intact, only minor modifications are introduced. Steering parameters are now better organized and final-state particles are stored in a common block [3] which is now becoming standard and used also in the programs KORALB [4] and KORALZ [5]. This facilitates the interface with other programs. In particular it allows for straightforward interface with PHOTOS $^{1}[6]$ general generator for QED corrections in decays. Importance sampling in the phase-space integration which is necessary to facilitate the generation of decay modes with more complicated resonance structure, are also introduced.

The present work is the third paper on the TAUOLA library. It was published originally in ref. [1] and subsequently updated for leptonic $\tau$-decay modes in ref. [7].

\footnotetext{
${ }^{1}$ PHOTOS version 1.06 or higher should be used.
} 


\section{PHASE SPACE AND MATRIX ELEMENTS}

Before we discuss in detail all the improvements made for this version of TAUOLA, let us recall some conventions and definitions of ref. [1].

The matrix element for the semileptonic decay $\tau \rightarrow \nu_{\tau}+X$ is written in the form:

$$
\mathcal{M}=\frac{G}{\sqrt{2}} \bar{u}(N) \gamma^{\mu}\left(v+a \gamma_{5}\right) u(P) J_{\mu}
$$

where $J_{\mu} \equiv\left\langle X\left|V_{\mu}-A_{\mu}\right| 0\right\rangle$ denotes the matrix element of the $V-A$ current relevant for the specific final state $X$. The current $J_{\mu}$ depends in general on the momenta of all hadrons and their polarizations. $N$ and $P$ denote, respectively, the four-momenta of the $\tau$ neutrino and the decaying $\tau$.

The squared matrix element for the decay of a $\tau$ with spin $s$ reads

$$
\begin{aligned}
|\mathcal{M}|^{2} & =G^{2} \frac{v^{2}+a^{2}}{2}\left(\omega+H_{\mu} s^{\mu}\right) \\
\omega & =P^{\mu}\left(\Pi_{\mu}-\gamma_{v a} \Pi_{\mu}^{5}\right) \\
H_{\mu} & =\frac{1}{M}\left(M^{2} g_{\mu}^{\nu}-P_{\mu} P^{\nu}\right)\left(\Pi_{\nu}^{5}-\gamma_{v a} \Pi_{\nu}\right)
\end{aligned}
$$

with

$$
\begin{aligned}
\Pi_{\mu} & =2\left(\left(J^{*} \cdot N\right) J_{\mu}+(J \cdot N) J_{\mu}^{*}-\left(J^{*} \cdot J\right) N_{\mu}\right), \\
\Pi^{5 \mu} & =2 \operatorname{Im} \epsilon^{\mu \nu \rho \sigma} J_{\nu}^{*} J_{\rho} N_{\sigma}, \\
\gamma_{v a} & =-\frac{2 v a}{v^{2}+a^{2}} .
\end{aligned}
$$

$\left(\gamma_{v a}=1\right.$ in the Standard Model). For a $\tau$-neutrino mass $m \neq 0$ and $v^{2} \neq a^{2}$ one has to add the following terms

$$
\begin{aligned}
\hat{\omega} & =2 \frac{v^{2}-a^{2}}{v^{2}+a^{2}} m M\left(J^{*} \cdot J\right), \\
\hat{H}^{\mu} & =-2 \frac{v^{2}-a^{2}}{v^{2}+a^{2}} m \operatorname{Im} \epsilon^{\mu \nu \rho \sigma} J_{\nu}^{*} J_{\rho} P_{\sigma}
\end{aligned}
$$

to $\omega$ and $H_{\mu}$, respectively. To obtain the polarimeter vector $h$ in the $\tau$ rest frame it is again sufficient to calculate the space components of $h_{\mu}=\left(H_{\mu}+\hat{H}_{\mu}\right) /(\omega+\hat{\omega})$ and set $h_{0}=0$ in the $\tau$ rest frame.

For $\tau^{+}$decays all momenta in $J_{\mu}$ are to be read as the momenta of the antiparticles and the terms proportional to $\gamma_{v a}$ reverse their signs. For the final states $X$, which receive contributions from hadronic vector and axial vector currents at the same time, the relative sign of the two contributions is reversed.

The partial decay rate for the channels with four particles in the final state reads

$$
2 M \Gamma_{X}=G^{2} \frac{v^{2}+a^{2}}{2} \int d \operatorname{Lips}\left(Q ; q_{i}, N\right)\left(\omega+\hat{\omega}+\left(H_{\mu}+\hat{H}_{\mu}\right) s^{\mu}\right) .
$$

As in ref. [1] we omit the spin-dependent part in the first step of the generation. 
The algorithm for events with one up to three mesons has been described in detail in ref. [1]. Using a more compact notation with $q_{4}=N$ and $q_{i}^{2}=m_{i}^{2}$ the phase space is rewritten as follows

$$
\begin{gathered}
d \operatorname{Lips}\left(P ; q_{1}, q_{2}, q_{3}, q_{4}\right)= \\
\frac{1}{2^{17} \pi^{8}} \int_{Q_{\text {min }}^{2}}^{Q_{\text {max }}^{2}} d Q^{2} \int_{M_{2, \text { min }}^{2}}^{M_{2, \text { max }}^{2}} d M_{2}^{2} \int d \Omega_{4} \frac{\lambda^{1 / 2}\left(M^{2}, Q^{2}, m_{4}^{2}\right)}{M^{2}} \int d \Omega_{3} \frac{\lambda^{1 / 2}\left(Q^{2}, m_{3}^{2}, M_{2}^{2}\right)}{Q^{2}} \int d \Omega_{2} \frac{\lambda^{1 / 2}\left(M_{2}^{2}, m_{2}^{2}, m_{1}^{2}\right)}{M_{2}^{2}}
\end{gathered}
$$

where

$$
\begin{aligned}
& M_{2}^{2}=\left(q_{1}+q_{2}\right)^{2}, \quad Q^{2} \quad=\left(q_{1}+q_{2}+q_{3}\right)^{2}, \\
& Q_{\min }=m_{1}+m_{2}+m_{3}, Q_{\max }=M-m_{4} \text {, } \\
& M_{2, \min }=m_{1}+m_{2}, \quad M_{2, \max }=Q-m_{3},
\end{aligned}
$$

$d \Omega_{4}=d \cos \theta_{4} d \phi_{4}$ is the solid-angle element of $\overrightarrow{q_{4}}$ (the momentum of $\nu_{\tau}$ ) in the rest frame of $\tau(P), d \Omega_{3}=d \cos \theta_{3} d \phi_{3}$ is the solid-angle element of $\overrightarrow{q_{3}}$ in the rest frame of $\left(q_{1}^{\mu}+q_{2}^{\mu}+q_{3}^{\mu}\right)$, and finally $d \Omega_{2}=d \cos \theta_{2} d \phi_{2}$ is the solid-angle element of $\overrightarrow{q_{2}}$ in the rest frame of $\left(q_{1}^{\mu}+q_{2}^{\mu}\right)$.

With the additional change of the variables

$$
\begin{array}{llrl}
\cos \theta_{2} & =-1+2 x_{1}, & \phi_{2} & =2 \pi x_{2}, \\
\cos \theta_{3} & =-1+2 x_{3}, & \phi_{3} & =2 \pi x_{4}, \\
\cos \theta_{4} & =-1+2 x_{5}, & \phi_{4} & =2 \pi x_{6}, \\
Q^{2} & =Q_{\text {min }}^{2}+\left(Q_{\max }^{2}-Q_{\text {min }}^{2}\right) x_{7}, & & \\
M_{2}^{2}=M_{2, \text { min }}^{2}+\left(M_{2, \text { max }}^{2}-M_{2, \text { min }}^{2}\right) x_{8} . &
\end{array}
$$

the integral (5) is transformed into a canonical form

$$
\int d \Gamma_{a}=\int_{0}^{1} \prod_{i=1}^{8} d x_{i} W\left(x_{1} \ldots x_{8}\right),
$$

where

$$
\begin{aligned}
W= & \frac{1}{2 M} G^{2} \frac{\left(v^{2}+a^{2}\right)}{2}(\omega+\hat{\omega}) J \\
= & \frac{1}{2 M} G^{2} \frac{\left(v^{2}+a^{2}\right)}{2}(\omega+\hat{\omega}) \frac{1}{2^{17} \pi^{8}} \frac{\lambda^{1 / 2}\left(M^{2}, m_{4}^{2}, Q^{2}\right)}{M^{2}} \frac{\lambda^{1 / 2}\left(Q^{2}, m_{3}^{2}, M_{2}^{2}\right)}{Q^{2}} \frac{\lambda^{1 / 2}\left(M_{2}^{2}, m_{2}^{2}, m_{1}^{2}\right)}{M_{2}^{2}} \\
& \times(4 \pi)^{3}\left(Q_{\max }^{2}-Q_{\min }^{2}\right)\left(M_{2, \max }^{2}-M_{2, \text { min }}^{2}\right) .
\end{aligned}
$$

The generation of events with four mesons plus a neutrino in the final state is very similar, the only difference being the dimension of the phase space. We use, as in the previous case, a compact notation with $q_{5}=N$ and $q_{i}^{2}=m_{i}^{2}$ :

$$
\begin{gathered}
d \operatorname{Lips}\left(P ; q_{1}, q_{2}, q_{3}, q_{4}, q_{5}\right)=\frac{1}{2^{23} \pi^{11}} \int_{Q_{\min }^{2}}^{Q_{\max }^{2}} d Q^{2} \int_{M_{3, \max }^{2}}^{M_{3, \max }^{2}} d M_{3}^{2} \int_{M_{2, \min }^{2}}^{M_{2, \max }^{2}} d M_{2}^{2} \\
\times \int d \Omega_{5} \frac{\lambda^{1 / 2}\left(M^{2}, Q^{2}, m_{5}^{2}\right)}{M^{2}} \int d \Omega_{4} \frac{\lambda^{1 / 2}\left(Q^{2}, M_{3}^{2}, m_{4}^{2}\right)}{Q^{2}} \int d \Omega_{3} \frac{\lambda^{1 / 2}\left(M_{3}^{2}, m_{3}^{2}, M_{2}^{2}\right)}{M_{3}^{2}} \int d \Omega_{2} \frac{\lambda^{1 / 2}\left(M_{2}^{2}, m_{2}^{2}, m_{1}^{2}\right)}{M_{2}^{2}},
\end{gathered}
$$


where

$$
\begin{array}{lll}
M_{2}^{2}=\left(q_{1}+q_{2}\right)^{2}, & M_{3}^{2}=\left(q_{1}+q_{2}+q_{3}\right)^{2}, \\
Q^{2}=\left(q_{1}+q_{2}+q_{3}+q_{4}\right)^{2}, & Q_{\text {min }}=m_{1}+m_{2}+m_{3}+m_{4}, \\
Q_{\max }=M-m_{5}, & M_{2, \min }=m_{1}+m_{2}, \\
M_{2, \max }=M_{3}-m_{3}, & M_{3, \min }=m_{1}+m_{2}+m_{3}, \\
M_{3, \max }=Q-m_{4},
\end{array}
$$

$d \Omega_{5}=d \cos \theta_{5} d \phi_{5}$ is the solid-angle element of $\overrightarrow{q_{5}}$ (the momentum of $\nu_{\tau}$ ) in the rest frame of $\tau(P), d \Omega_{4}=d \cos \theta_{4} d \phi_{4}$ is the solid-angle element of $\overrightarrow{q_{4}}$ in the $\left(q_{1}^{\mu}+q_{2}^{\mu}+q_{3}^{\mu}+q_{4}^{\mu}\right)$ rest frame, $d \Omega_{3}=d \cos \theta_{3} d \phi_{3}$ is the solid-angle element of $\overrightarrow{q_{3}}$ in the $\left(q_{1}^{\mu}+q_{2}^{\mu}+q_{3}^{\mu}\right)$ rest frame, and finally $d \Omega_{2}=d \cos \theta_{2} d \phi_{2}$ is the solid-angle element of $\overrightarrow{q_{2}}$ in the rest frame of $\left(q_{1}^{\mu}+q_{2}^{\mu}\right)$.

With the additional change of the variables

$$
\begin{array}{lll}
\cos \theta_{2}=-1+2 x_{1}, & \phi_{2}=2 \pi x_{2}, \\
\cos \theta_{3}=-1+2 x_{3}, & \phi_{3}=2 \pi x_{4}, \\
\cos \theta_{4}=-1+2 x_{5}, & \phi_{4}=2 \pi x_{6}, \\
\cos \theta_{5}=-1+2 x_{7}, & \phi_{5}=2 \pi x_{8}, \\
Q^{2}=Q_{\text {min }}^{2}+\left(Q_{\max }^{2}-Q_{\min }^{2}\right) x_{9}, & \\
M_{3}^{2}=M_{3, \text { min }}^{2}+\left(M_{3, \text { max }}^{2}-M_{3, \text { min }}^{2}\right) x_{10}, & \\
M_{2}^{2}=M_{2, \text { min }}^{2}+\left(M_{2, \text { max }}^{2}-M_{2, \text { min }}^{2}\right) x_{11} . &
\end{array}
$$

the integral is again transformed into a canonical form

$$
\int d \Gamma_{X}=\int_{0}^{1} \prod_{i=1}^{11} d x_{i} W\left(x_{1} \ldots x_{11}\right),
$$

where

$$
\begin{aligned}
W= & \frac{1}{2 M} G^{2} \frac{\left(v^{2}+a^{2}\right)}{2}(\omega+\hat{\omega}) J \\
= & \frac{1}{2 M} G^{2} \frac{\left(v^{2}+a^{2}\right)}{2}(\omega+\hat{\omega}) \\
& \times \frac{1}{2^{23} \pi^{11}} \frac{\lambda^{1 / 2}\left(M^{2}, m_{5}^{2}, Q^{2}\right)}{M^{2}} \frac{\lambda^{1 / 2}\left(Q^{2}, m_{4}^{2}, M_{3}^{2}\right)}{Q^{2}} \frac{\lambda^{1 / 2}\left(M_{3}^{2}, m_{3}^{2}, M_{2}^{2}\right)}{M_{3}^{2}} \frac{\lambda^{1 / 2}\left(M_{2}^{2}, m_{2}^{2}, m_{1}^{2}\right)}{M_{2}^{2}} \\
& \times(4 \pi)^{4}\left(Q_{\text {max }}^{2}-Q_{\text {min }}^{2}\right)\left(M_{2, \text { max }}^{2}-M_{2, \text { min }}^{2}\right)\left(M_{3, \text { max }}^{2}-M_{3, \text { min }}^{2}\right)
\end{aligned}
$$

These formulae are inefficient for a Monte Carlo algorithm if sharp peaks due to resonances are present. It is rather straightforward to resolve this if the resonance can be saturated by one of the Lorentz invariants used in phase-space generation ( $Q$ or $\left.M_{i}\right)$. Then, the term ${ }^{2}$

$$
Q^{2}=Q_{\min }^{2}+\left(Q_{\max }^{2}-Q_{\min }^{2}\right) x_{i}
$$

has to be replaced by

$$
\begin{array}{ll}
Q^{2} & =M_{R}^{2}+\Gamma_{R} M_{R} \tan \alpha, \\
\alpha & =\alpha_{\min }+\left(\alpha_{\max }-\alpha_{\min }\right) x_{i}, \\
Q_{\min / \max }^{2} & =M_{R}^{2}+\Gamma_{R} M_{R} \tan \alpha_{\min / \max },
\end{array}
$$

\footnotetext{
${ }^{2} \mathrm{Or}: \quad M_{i}^{2}=M_{i, \text { min }}^{2}+\left(M_{i, \text { max }}^{2}-M_{i, \min }^{2}\right) x_{i}$.
} 
and in the corresponding Jacobian factors [formulae (10) and (15)] the terms

$$
\left(Q_{\max }^{2}-Q_{\min }^{2}\right)
$$

have to be replaced by

$$
\frac{\left(Q^{2}-M_{R}^{2}\right)^{2}+\left(\Gamma_{R} M_{R}\right)^{2}}{M_{R} \Gamma_{R}}\left(\alpha_{\max }-\alpha_{\min }\right) .
$$

In more complicated cases, when it is not possible to localize all resonances in a single chain of the Lorentz-invariant variables $M_{i}, Q$ the $\mathrm{MC}$ algorithm has to be split into branches [8]. In the most general case, when $n$ different parametrizations of the phase space with different orderings of particles are in use, the differential rate of the decay into $X$ can be written as follows:

$$
\begin{aligned}
d \Gamma_{X}= & \sum_{\lambda=1}^{n} P_{\lambda} \int_{0}^{1} \prod_{i=1}^{m} d x_{i}\left[\sum_{\delta=1}^{n} P_{\delta} J_{\delta}^{-1}\left(q_{1}\left(\lambda, x_{i}\right), \ldots q_{k}\left(\lambda, x_{i}\right)\right)\right]^{-1} \\
& \times \frac{1}{2 M} G^{2} \frac{\left(v^{2}+a^{2}\right)}{2}(\omega+\hat{\omega}) .
\end{aligned}
$$

In the above formula the four-momenta $q_{i}\left(\lambda, x_{i}\right)$ are calculated from the random numbers $x_{i}$ according to the parametrization of the phase space of type $\lambda$. The Jacobians $J_{\delta}$ have to be calculated for all parametrizations of the phase space at the point $q_{i} ; P_{\lambda}$ denotes the probability of choosing the parametrization of type $\lambda$ in the generation, $\lambda$ thus takes ${ }^{3}$ a role of an additional discrete variable in the generation. Numerical values of probabilities $P_{\lambda}$ do not affect the final distributions, but only the efficiency of the generation. This is quite similar to the case of $M_{R}, \Gamma_{R}$ defined in formula (17).

This formal solution incorporates symmetrization over identical particles; the corresponding statistical factor has to be included explicitly.

Before we go into a discussion of the particular new decay modes, let us note that, for our tests, we will use $m_{\pi^{ \pm}}=m_{\pi^{0}}=0.1395 \mathrm{GeV}, m_{K^{ \pm}}=m_{K^{0}}=0.0 .493667 \mathrm{GeV}$. In the case of the demonstration deck (and demonstration output) we will return to the numerical values defined in ref. [1].

\section{HADRONIC CURRENTS FOR $2 K \pi, K 2 \pi, 3 \pi$ and $\eta 2 \pi$}

The hadronic currents producing three scalar particles $x_{1}\left(p_{1}\right), x_{2}\left(p_{2}\right), x_{3}\left(p_{3}\right)$ have been separated into three parts. We distinguish a chiral, a scalar, and an anomalous part.

1. Let us begin with the chiral part, which is linear in the transverse part of the momenta of scalar particles. This structure $[9,10,11,12,13]$ is obtained from the chiral lagrangian and we have implemented two- and three-body resonances in the form factors $F_{1}, F_{2}, F_{3}$, which are normalized to 1 in the limit of vanishing momenta. The form factor $F_{3}$ is redundant and could have been dropped without any loss of generality. For the axial vector channel we consider only the lowest-lying resonance and couple it with full strength to the weak gauge boson.

\footnotetext{
${ }^{3}$ But not $\delta$.
} 
2. A scalar contribution $\left(F_{4}\right)$ [12], which has to vanish in the chiral limit. Up to now we only have included the case of an exited $\pi^{\prime}(1300)$ as additional option (default is $F_{4}=0$ ). The couplings have been fixed by using a quark-model calculation of ref. [14].

3. The contribution of the Wess-Zumino anomaly for $S U(3)_{L} \times S U(3)_{R}$ has been calculated in $[15,16]$. These terms induce a contribution of the weak vector current to the decay into three mesons. Again resonances (not only the lowest one!) have been implemented in the form factor $F_{5}$ and the condition that $F_{5}$ reduces to unity in the chiral limit has been maintained.

Using Lorentz invariance the hadronic current can be cast into the following form

$$
\begin{aligned}
J^{\mu}=N \quad & \left\{T_{\nu}^{\mu}\left[c_{1}\left(p_{2}-p_{3}\right)^{\nu} F_{1}+c_{2}\left(p_{3}-p_{1}\right)^{\nu} F_{2}+c_{3}\left(p_{1}-p_{2}\right)^{\nu} F_{3}\right]\right. \\
& \left.+c_{4} q^{\mu} F_{4}-\frac{i}{4 \pi^{2} f_{\pi}^{2}} c_{5} \epsilon_{. \nu \rho \sigma}^{\mu} p_{1}^{\nu} p_{2}^{\rho} p_{3}^{\sigma} F_{5}\right\},
\end{aligned}
$$

where $T_{\mu \nu}=g_{\mu \nu}-q_{\mu} q_{\nu} / q^{2}$ is the transverse projector, $q=p_{1}+p_{2}+p_{3}$ and $f_{\pi}=0.0933$ $\mathrm{GeV}$. Note again that one of the form factors $F_{1,2,3}$ is redundant. The constants $N, c_{i}$ and the form factors $F_{i} \equiv F_{i}\left(q^{2}, s_{1}, s_{2}, s_{3}\right)$ depend on the particular hadronic final state. The Lorentz invariants are related to the particle momenta through $s_{i}=\left(p_{j}+p_{k}\right)^{2}$, where $i \neq j \neq k \neq i$. Not all of the Lorentz invariants are independent. In our applications we choose to order the particles in such a way that $F_{3}$ can be dropped conveniently and eventual resonances occur in the kinematical variables $s_{1}, s_{2}$ or $q^{2}$.

In the following two tables the numerical constants and form-factors are collected. Note that the ordering of the particles in the tables is such that the first and second entries have momentum $p_{1}$ and $p_{2}$, respectively ${ }^{4}$.

\begin{tabular}{|r|r|r|r|r|r|r|}
\hline Decay mode & $N$ & $c_{1}$ & $c_{2}$ & $c_{3}$ & $c_{4}$ & $c_{5}$ \\
\hline$\pi^{-} \pi^{-} \pi^{+}$ & $\frac{\cos \theta_{c}}{f_{\pi}}$ & $\frac{2 \sqrt{2}}{3}$ & $-\frac{2 \sqrt{2}}{3}$ & 0 & 0 & 0 \\
$K^{-} \pi^{-} K^{+}$ & $\frac{\cos \theta_{c}}{f_{\pi}}$ & $-\frac{\sqrt{2}}{3}$ & $\frac{\sqrt{2}}{3}$ & 0 & 0 & $\sqrt{2}$ \\
$K^{0} \pi^{-} \bar{K}^{0}$ & $\frac{\cos \theta_{c}}{f_{\pi}}$ & $-\frac{\sqrt{2}}{3}$ & $\frac{\sqrt{2}}{3}$ & 0 & 0 & $-\sqrt{2}$ \\
$K^{-} \pi^{0} K^{0}$ & $\frac{\cos \theta_{c}}{f_{\pi}}$ & 0 & -1 & 0 & 0 & 0 \\
$\pi^{0} \pi^{0} K^{-}$ & $\frac{\sin \theta_{c}}{\sqrt{2} f_{\pi}}$ & $\frac{1}{3 \sqrt{2}}$ & $-\frac{1}{3 \sqrt{2}}$ & 0 & 0 & 0 \\
$K^{-} \pi^{-} \pi^{+}$ & $\frac{\sin \theta_{c}}{f_{\pi}}$ & $-\frac{\sqrt{2}}{3}$ & $\frac{\sqrt{2}}{3}$ & 0 & 0 & $-\sqrt{2}$ \\
$\pi^{-} \bar{K}^{0} \pi^{0}$ & $\frac{\sin \theta_{c}}{f_{\pi}}$ & 0 & -1 & 0 & 0 & -2 \\
$\pi^{-} \pi^{0} \eta$ & $\frac{\cos \theta_{c}}{f_{\pi}}$ & 0 & 0 & 0 & 0 & $\sqrt{\frac{2}{3}}$ \\
\hline
\end{tabular}

We prefer to present normalization and form factors separately in order to remind the reader that the former are obtained from the chiral Lagrangian.

The parametrization of the form factors in the next table is taken from [12].

\footnotetext{
${ }^{4}$ As an additional option we allow for a scalar contribution in the three-pion channel as discussed below.
} 


\begin{tabular}{|r|r|r|r|r|}
\hline Dec. mod. & $F_{1}\left(q^{2}, s_{1}, s_{2}\right)$ & $F_{2}\left(q^{2}, s_{2}, s_{1}\right)$ & $F_{4}\left(q^{2}, s_{1}, s_{2}, s_{3}\right)$ & $F_{5}\left(q^{2}, s_{1}, s_{2}\right)$ \\
\hline$\pi^{-} \pi^{-} \pi^{+}$ & $B_{a_{1}}\left(q^{2}\right) B_{\rho}\left(s_{1}\right)$ & $B_{a_{1}}\left(q^{2}\right) B_{\rho}\left(s_{2}\right)$ & 0 & 0 \\
$K^{-} \pi^{-} K^{+}$ & $B_{a_{1}}\left(q^{2}\right) B_{K^{*}}\left(s_{1}\right)$ & $B_{a_{1}}\left(q^{2}\right) B_{\rho}\left(s_{2}\right)$ & 0 & $B_{\rho}^{1}\left(q^{2}\right) F_{K^{*}, \rho}\left(s_{1}, s_{2}, \alpha\right)$ \\
$K^{0} \pi^{-} \bar{K}^{0}$ & $B_{a_{1}}\left(q^{2}\right) B_{K^{*}}\left(s_{1}\right)$ & $B_{a_{1}}\left(q^{2}\right) B_{\rho}\left(s_{2}\right)$ & 0 & $B_{\rho}^{1}\left(q^{2}\right) F_{K^{*}, \rho}\left(s_{1}, s_{2}, \alpha\right)$ \\
$K^{-} \pi^{0} K^{0}$ & 0 & $B_{a_{1}}\left(q^{2}\right) B_{\rho}\left(s_{2}\right)$ & 0 & 0 \\
$\pi^{0} \pi^{0} K^{-}$ & $B_{K_{1}}\left(q^{2}\right) B_{K^{*}}\left(s_{1}\right)$ & $B_{K_{1}}\left(q^{2}\right) B_{K^{*}}\left(s_{2}\right)$ & 0 & 0 \\
$K^{-} \pi^{-} \pi^{+}$ & $B_{K_{1}}\left(q^{2}\right) B_{\rho}\left(s_{1}\right)$ & $B_{K_{1}}\left(q^{2}\right) B_{K^{*}}\left(s_{2}\right)$ & 0 & 0 \\
$\pi^{-} \bar{K}^{0} \pi^{0}$ & 0 & $B_{K_{1}}\left(q^{2}\right) B_{\rho}\left(s_{2}\right)$ & 0 & $B_{K^{*}}\left(q^{2}\right) F_{K^{*}, \rho}\left(s_{2}, s_{1}, \alpha\right)$ \\
$\pi^{-} \pi^{0} \eta$ & 0 & 0 & 0 & $B_{K^{*}}\left(q^{2}\right) F_{K^{*}, \rho}\left(s_{1}, s_{2}, \alpha\right)$ \\
& 0 & 0 & $B_{\rho}^{1}\left(q^{2}\right) B_{\rho}\left(s_{1}\right)$ \\
\hline
\end{tabular}

We have defined $B_{a_{1}}(s)$ and $B_{\rho}(s)$ as in eqs. (3.34) and (3.44) of ref. [1]. Lacking experimental information, a constant width is taken for the $1^{+}$resonance $K_{1}$. For all other two-body resonances we employ an energy-dependent width as dictated by the $p$-wave phase space. Furthermore the following parametrization for the Breit-Wigner amplitudes are used:

$$
\begin{aligned}
& \Gamma_{R}(s)=\Gamma_{R} \frac{m_{R}^{2}}{s}\left(\frac{p(s)}{p\left(M_{R}^{2}\right)}\right)^{\frac{3}{2}}, \\
& \widehat{B W}_{i}\left(s, m_{i}, \Gamma_{i}\right)=\frac{m_{i}^{2}-i m_{i} \Gamma_{i}}{\left(m_{i}^{2}-s-i m_{i} \Gamma_{i}\right)}, \\
& B W_{i}\left(s, m_{i}, \Gamma_{i}\right)=\frac{m_{i}^{2}}{\left(m_{i}^{2}-s-i \sqrt{s} \Gamma_{i}(s)\right)}, \\
& B_{K_{1}}(s) \quad=B \widehat{W}_{K_{1}}\left(s, m_{K_{1}}, \Gamma_{K_{1}}\right) \text {, } \\
& m_{1} \quad=1.402 \mathrm{GeV}, \quad \Gamma_{1}=0.174 \mathrm{GeV} \text {, } \\
& \begin{aligned}
B_{K^{*}}(s) & =B W\left(s, m_{K^{*}}, \Gamma_{K^{*}}(s)\right), & & \\
m_{K^{*}} & =0.892 \mathrm{GeV}, & \Gamma_{K^{*}} & =0.0513 \mathrm{GeV},
\end{aligned} \\
& F_{K^{*}, \rho}\left(s_{i}, s_{j}, \alpha\right)=\left[\alpha B_{K^{*}}\left(s_{i}\right)+B_{\rho}\left(s_{j}\right)\right] /(1+\alpha), \\
& B_{\rho}^{1}(s)=\frac{1}{1+\beta+\delta}\left\{B W\left(s, \rho^{\prime \prime}, \Gamma_{\rho^{\prime \prime}}(s)\right)+\beta B W\left(s, \rho^{\prime}, \Gamma_{\rho^{\prime}}(s)\right)+\delta B W\left(s, \rho, \Gamma_{\rho}(s)\right)\right\}, \\
& \delta=-26, m_{\rho}=0.773 \mathrm{GeV}, \Gamma_{\rho}=0.145 \mathrm{GeV}, \\
& \beta=6.5, \quad m_{\rho^{\prime}}=1.5 \mathrm{GeV}, \quad \Gamma_{\rho^{\prime}}=0.220 \mathrm{GeV}, \\
& m_{\rho^{\prime \prime}}=1.75 \mathrm{GeV}, \quad \Gamma_{\rho^{\prime \prime}}=0.120 \mathrm{GeV} \text {, }
\end{aligned}
$$

A reasonable fit of the $\tau$ decay into $K^{-} \pi^{-} \pi^{+}$is obtained when taking $\alpha=-0.2$ [12]. The $B_{\rho}^{1}(s)$ parametrization [17] is taken from $e^{+}+e^{-} \rightarrow \eta \pi \pi$ data and is needed to reproduce the measured decay width into $\eta \pi^{-} \pi^{0}$. It is used only in the Cabibbo-allowed vector form factor $F_{5}$.

As an additional (but not default) option we allow for a scalar contribution in the three-pion channel. In this case $c_{4}=1$ and the form factor for the scalar contribution is 
given by:

$$
\begin{array}{ll}
F_{4}\left(s, s_{1}, s_{2}\right)= & \frac{g_{\pi^{\prime} \rho \pi} g_{\rho \pi \pi} f_{\pi^{\prime}} f_{\pi}}{m_{\rho}^{4} m_{\pi^{\prime}}^{2}} B_{\pi^{\prime}}\left(s, m_{\pi^{\prime}}, \Gamma_{\pi^{\prime}}(s)\right)\left(s_{1}\left(s_{2}-s_{3}\right) B_{\rho}\left(s_{1}\right)+(1 \leftrightarrow 2)\right), \\
& \Gamma_{\pi^{\prime}}(s)=\Gamma_{\pi^{\prime}} \frac{m_{\pi^{\prime}}^{2}}{s} \frac{\left\{\left(s-m_{\rho}^{2}-m_{\pi}^{2}\right)^{2}-4 m_{\rho}^{2} m_{\pi}^{2}\right\}^{5 / 2} m_{\pi^{\prime}}^{5}}{\left\{\left(m_{\pi^{\prime}}^{2}-m_{\rho}^{2}-m_{\pi}^{2}\right)^{2}-4 m_{\rho}^{2} m_{\pi}^{2}\right\}^{5 / 2}{ }_{s^{5 / 2}}}, \\
m_{\pi^{\prime}}=1.3 \mathrm{GeV}, \quad & \Gamma_{\pi^{\prime}}=0.3 \mathrm{GeV}, \\
f_{\pi^{\prime}}=0.02 \mathrm{GeV}, \quad & g_{\pi^{\prime} \rho \pi}=5.8, \\
g_{\rho \pi \pi}=6.08 . &
\end{array}
$$

Some comments are in order:

We have introduced energy-dependent $K^{*}$ and $\pi^{\prime}$ widths. However, we do not distinguish between the mass of the charged and neutral kaons and pions in the $K^{*}$ decay. For the $\pi^{\prime}$ width we have used a simplified dependence by assuming the dominant decay channel to be $\pi^{\prime} \rightarrow \rho \pi$. Finally we recall that the parameters for the $\pi^{\prime}$ contribution originate from a quark-model calculation [14], not from experiment.

On the numerical side, in order to facilitate the generation, we have introduced importance sampling in both the $M_{2}^{2}$ and $Q^{2}$ variables. For $M_{2}^{2}$ we use the mass and width of one of the secondary resonances, depending on the particular decay mode; for $Q^{2}$ it was more convenient to fit the shape of the distribution with some "effective" resonance parameters. As these are just technical parameters, which cancel completely in the final results, this solution is fully justified. To incorporate in pre-sampling the complete peak structure we have applied the solution of eq. (20). In two channels of the generation the variable $M_{2}^{2}$ corresponds respectively to $s_{1}$ and $s_{2}$. The channel without resonance pre-sampling of $M_{2}^{2}$ is also included.

In the following table we collect branching ratios (in $\Gamma_{e}$ units) as obtained in the demonstration output. They are calculated from the form factors and parameters of this paper, in addition to those already defined in [1]. As a cross check, we compare in this table the results of Monte Carlo and of the semi-analytical calculation [12]. For $\alpha=-0.2$ we obtain:

\begin{tabular}{|l|l|l|}
\hline Decay mode & Monte Carlo result & Semi-analytical result \\
\hline$\pi^{-(0)} \pi^{-(0)} \pi^{+(-)}$ & $0.71143 \pm 0.1 \%$ & $2 \times 0.356$ \\
$K^{-} \pi^{-} K^{+}$ & $0.00606 \pm 0.1 \%$ & 0.0061 \\
$K^{0} \pi^{-} \bar{K}^{0}$ & $0.00606 \pm 0.1 \%$ & 0.0061 \\
$K^{-} \pi^{0} K^{0}$ & $0.00053 \pm 0.5 \%$ & 0.0005 \\
$\pi^{0} \pi^{0} K^{-}$ & $0.00599 \pm 0.1 \%$ & 0.0060 \\
$K^{-} \pi^{-} \pi^{+}$ & $0.03147 \pm 0.1 \%$ & 0.0316 \\
$\pi^{-} \bar{K}^{0} \pi^{0}$ & $0.03237 \pm 0.1 \%$ & 0.0324 \\
$\pi^{-} \pi^{0} \eta$ & $0.01082 \pm 0.1 \%$ & 0.0108 \\
\hline
\end{tabular}

Inclusion of the scalar form factor would increase the $3 \pi$ rate by $0.00935 \pm 0.1 \%$, in agreement with the result $2 \times 0.0046$ from ref. [12]. 
Had we used the parametrization $B_{\rho}\left(q^{2}\right)$ instead of $B_{\rho}^{1}\left(q^{2}\right)$ for the form factor in the anomalous part, the $\eta \pi^{0} \pi^{-}$branching ratio would have been too small $(0.0004)$, as compared with present experimental results. This situation is therefore cured by the use of the form factor $B_{\rho}^{1}\left(q^{2}\right)$, which includes a $\rho^{\prime}$ and a $\rho^{\prime \prime}[12,17]$.

\section{$\omega$-CONTRIBUTION TO THE $\pi^{-}\left(p_{3}\right) \pi^{0}\left(p_{2}\right) \gamma\left(p_{1}\right)$ DECAY}

Since the $\omega$ has a sizeable decay into $\pi^{0} \gamma$, we expect some contribution to $\pi^{-} \pi^{0} \gamma$, from $\tau \rightarrow \omega \pi \nu_{\tau}$. This is of importance if a precise determination of the parameters of the $\rho$-resonance is to be performed in $\tau$ decays.

The only difference, with respect to the hadronic current described in the previous chapter, is that the photon can be produced in two different polarization states $\left(\epsilon_{\mu}^{1,2}\right)$ and the appropriate sum has to be performed. The current is given by the following expression:

$$
\begin{aligned}
J_{\mu}^{1,2}=e \cos \theta_{c} T & \left\{\epsilon_{\mu}^{1,2}\left[m_{\pi}^{2}\left(p_{3} \cdot p_{1}\right)-\left(p_{2} \cdot p_{1}\right)\left(\left(p_{3} \cdot p_{2}\right)-\left(p_{3} \cdot p_{1}\right)\right)\right]\right. \\
& -p_{2 \mu}\left[\left(p_{2} \cdot \epsilon^{1,2}\right)\left(p_{3} \cdot p_{1}\right)-\left(p_{3} \cdot \epsilon^{1,2}\right)\left(p_{2} \cdot p_{1}\right)\right] \\
+ & \left.p_{1 \mu}\left[\left(p_{2} \cdot \epsilon^{1,2}\right)\left(p_{3} \cdot p_{2}\right)-\left(p_{3} \cdot \epsilon^{1,2}\right)\left(m_{\pi}^{2}+\left(p_{2} \cdot p_{1}\right)\right)\right]\right\},
\end{aligned}
$$

where

$$
\begin{aligned}
& T=F\left(q^{2}\right) F(0) \frac{1}{\sqrt{2}\left(m_{\omega}^{2}-i m_{\omega} \Gamma_{\omega}\right)} \widehat{B W}\left(s_{1}, m_{\omega}, \Gamma_{\omega}\right) \\
& F(s)=F_{\rho} g_{\rho \omega \pi}\left(\frac{1}{m_{\rho}^{2}-i m_{\rho} \Gamma_{\rho}} \widehat{B W}\left(s, m_{\rho} \Gamma_{\rho}\right)+\sigma \frac{1}{m_{\rho^{\prime}}^{2}-i m_{\rho^{\prime}} \Gamma_{\rho^{\prime}}} \widehat{B W}\left(s, m_{\rho^{\prime}} \Gamma_{\rho^{\prime}}\right)\right) \\
& F_{\rho}=0.266 m_{\rho}^{2} ; \quad \sigma=-0.1 ; g_{\rho \omega \pi}=12.924 \mathrm{GeV}^{-1} ;
\end{aligned}
$$

Note that the value of $g_{\rho \omega \pi}$ is obtained from the $\omega \rightarrow \pi \gamma$ decay with $\sigma=-0.1$. For other values of $\sigma$ the coupling constant can be obtained from

$$
\Gamma(\omega \rightarrow \pi \gamma)=\frac{m_{\omega}^{3} F_{\rho}^{2} g_{\rho \omega \pi}^{2} \alpha}{48 m_{\rho}^{2}\left(m_{\rho}^{2}+\Gamma_{\rho}^{2}\right)}\left(1-\frac{m_{\pi}^{2}}{m_{\omega}^{2}}\right)^{3}\left|1+\sigma \frac{m_{\rho}}{m_{\rho^{\prime}}} \frac{m_{\rho}-i \Gamma_{\rho}}{m_{\rho^{\prime}}-i \Gamma_{\rho^{\prime}}}\right|^{2}
$$

For the numerical input we use some parameters from the $\tau \rightarrow \rho \nu$ decay channel, that is: $m_{\rho}=0.773 \mathrm{GeV}, \Gamma_{\rho}=0.145 \mathrm{GeV}$, and some new parameters: $m_{\rho^{\prime}}=1.7 \mathrm{GeV}$, $\Gamma_{\rho^{\prime}}=0.26 \mathrm{GeV}$, and $m_{\omega}=0.782 \mathrm{GeV}, \Gamma_{\omega}=0.0085 \mathrm{GeV}$.

Our program yields

$$
\frac{\Gamma\left(\tau^{-} \rightarrow \nu_{\tau} \pi^{-} \omega\left(\rightarrow \pi^{0} \gamma\right)\right)}{\Gamma_{e}}=0.003135 \pm 0.1 \%
$$

We have checked that our program reproduces the semi-analytical result of ref. [19] $\left(0.3297 \times \cos ^{2} \theta_{c} \times 10^{-2}\right)$ with $0.1 \%$ accuracy, and that our generated $d \Gamma / d Q^{2}$ spectrum 
is indistinguishable from that available from ref. [19]. We have also checked, that up to $0.1 \%$, in the limit $m_{\pi} \rightarrow 0$, our program reproduces the corresponding semi-analytical result for the ratio (30) as well.

To facilitate generation we have introduced importance sampling both in the $M_{2}^{2}$ and $Q^{2}$ variables. For $M_{2}^{2}$ we have used the $\omega$ mass and width, while for $Q^{2}$ it was more convenient to fit the shape of the distribution with some "effective" resonance parameters. This solution is fully justified as these are pure technical parameters and cancel out completely, in the final results.

\section{HADRONIC CURRENTS for $4 \pi$}

There are two $4 \pi$ final states in $\tau$ decay:

i) $\tau \rightarrow \nu \pi_{-}\left(p_{1}\right) \pi_{0}\left(p_{2}\right) \pi_{0}\left(p_{3}\right) \pi_{0}\left(p_{4}\right)$

ii) $\tau \rightarrow \nu \pi_{+}\left(p_{1}\right) \pi_{0}\left(p_{2}\right) \pi_{-}\left(p_{3}\right) \pi_{-}\left(p_{4}\right)$.

In the first case the chiral current [9] can again be modified by resonances. For the two-meson resonances we use $B_{\rho}\left(s_{i}\right)$ as defined in (3.44) of [1]. For the time being the resonance structure in $Q^{2}$ is described by the same function. We are aware that higher resonances should be included eventually, especially once the new $e^{+}+e^{-} \rightarrow 4 \pi$ data are available to allow for a parametrization:

$$
\begin{aligned}
& J_{\mu}=\frac{2 \sqrt{3}}{f_{\pi}^{2}} \cos \theta_{c} B_{\rho}\left(q^{2}\right) \sum_{k=2}^{4} A_{\mu \nu}^{k} B_{\rho}\left(s_{k}\right)\left(p_{k}-p_{1}\right)^{\nu}, \\
& s_{k}=\left(p_{k}+p_{1}\right)^{2}, \quad k=2,3,4, \quad q=q_{1}+q_{2}+q_{3}+q_{4}, \\
& A_{\mu \nu}^{k}=g_{\mu \nu}-\sum_{l=2, l \neq k}^{4} \frac{\left(q-2 p_{l}\right)_{\mu}\left(q-p_{l}\right)_{\nu}}{\left(q-p_{l}\right)^{2}} .
\end{aligned}
$$

In the second case the current can be written as a sum over a part that originates from the chiral Lagrangian (with resonances) and an anomalous part ( $\rho \omega \pi$-coupling) [20]:

$$
J_{\mu}=J_{\mu}^{I}+J_{\mu}^{\omega}
$$

where

$$
\begin{aligned}
& J_{\mu}^{I}=\frac{2 \sqrt{3}}{f_{\pi}^{2}} \cos \theta_{c} B_{\rho}\left(q^{2}\right) \sum_{k=2}^{4} R_{k} A_{\mu \nu}^{k} B_{\rho}\left(s_{k}\right)\left(p_{k}-p_{1}\right)^{\nu}, \\
& R_{k}=1 \text { for } k=3,4, \quad R_{k}=-2 \text { for } k=2
\end{aligned}
$$

and

$$
\begin{aligned}
J_{\mu}^{\omega}= & \left(\frac{1}{m_{\rho}^{2}-i m_{\rho} \Gamma_{\rho}} \widehat{B W}_{\rho}\left(q^{2}, m_{\rho}, \Gamma_{\rho}\right)+\sigma \frac{1}{m_{\rho^{\prime}}^{2}-i m_{\rho^{\prime}} \Gamma_{\rho^{\prime}}} \widehat{B W}_{\rho^{\prime}}\left(q^{2}, m_{\rho^{\prime}}, \Gamma_{\rho^{\prime}}\right)\right) \\
& \cos \theta_{c} G_{\omega 3 \pi} g_{\rho \omega \pi} F_{\rho}\left\{\frac{1}{m_{\omega}^{2}-i m_{\omega} \Gamma_{\omega}} \widehat{B W}_{\omega}\left(\left(q-p_{4}\right)^{2}, m_{\omega}, \Gamma_{\omega}\right)\right. \\
& {\left[p_{3 \mu}\left(\left(q-p_{4}\right) \cdot p_{2} p_{1} \cdot p_{4}-\left(q-p_{4}\right) \cdot p_{1} p_{2} \cdot p_{4}\right)\right.} \\
& +p_{2 \mu}\left(\left(q-p_{4}\right) \cdot p_{1} p_{3} \cdot p_{4}-\left(q-p_{4}\right) \cdot p_{3} p_{1} \cdot p_{4}\right) \\
& \left.p_{1 \mu}\left(\left(q-p_{4}\right) \cdot p_{3} p_{2} \cdot p_{4}-\left(q-p_{4}\right) \cdot p_{2} p_{3} \cdot p_{4}\right)\right] \\
& +(3 \leftrightarrow 4)\} .
\end{aligned}
$$


Note that we have fixed the relative sign of the "chiral" and anomalous parts in the chiral limit. We use $G_{\omega 3 \pi}=1476 \mathrm{GeV}^{-3}$, determined from the present data [3] on $\omega$. The differential width is given by

$$
\frac{d \Gamma}{d s_{1} d s_{2}}=\frac{G_{\omega 3 \pi}^{2}}{384(2 \pi)^{3} m_{\omega}^{3}}\left(\left(m_{\omega}^{2}+3 m_{\pi}^{2}\right) s_{1} s_{2}-\left(s_{1} s_{2}^{2}+s_{1}^{2} s_{2}\right)-m_{\pi}^{2}\left(m_{\omega}^{2}-m_{\pi}^{2}\right)^{2}\right) .
$$

The total width for massless pions simplifies to

$$
\Gamma_{\omega}=\frac{1}{90(16 \pi)^{3}} G_{\omega 3 \pi}^{2} m_{\omega}^{7} .
$$

The remaining constants are already defined above.

To speed-up the generation we have introduced importance sampling in both variables $M_{3}^{2}$ and $Q^{2}$. There are three parallel chains of generation. In two cases the $M_{3}^{2}$ corresponds to the $\omega$ resonance and thus we have used the $\omega$ mass and width for pre-sampling. In the third case $M_{3}^{2}$ is generated without pre-sampling. For $Q^{2}$ it was more convenient to fit the shape of the distribution with some "effective" resonance parameters. These are just technical parameters and we checked numerically that they cancel completely in the final results.

In the following table, we collect numerical results calculated by the program in the normal mode of operation. In the second column the results are obtained with $m_{\pi}=0$ and all $B W(x, m, \Gamma)=1$. In the third column the result of the analytical calculation is given for this idealized case $^{5}$. The analytical results are

$$
\begin{gathered}
\frac{\Gamma\left(\pi^{-} 3 \pi^{0}\right)}{\Gamma_{e}}=\frac{\cos ^{2} \theta_{c}}{15}\left(\frac{m_{\tau}}{2 \pi f_{\pi}}\right)^{4} \frac{1}{256}\left(\pi^{2}-\frac{437}{48}\right), \\
\frac{\Gamma\left(2 \pi^{-} \pi^{+} \pi^{0}\right)}{\Gamma_{e}}=\frac{\cos ^{2} \theta_{c}}{15}\left(\frac{m_{\tau}}{2 \pi f_{\pi}}\right)^{4} \frac{1}{128}\left(\frac{1261}{120}-\pi^{2}\right) .
\end{gathered}
$$

Good agreement is found.

We also compare the result for the $\pi \omega$ mode in the massless case and zero-width approximation, again; good agreement is obtained.

\begin{tabular}{|l|l|l|l|}
\hline Decay mode & MC result & $\begin{array}{l}\text { MC result } \\
\left(m_{\pi}=0, B W=1\right)\end{array}$ & $\begin{array}{l}\text { analytical } \\
\text { result }\end{array}$ \\
\hline$\frac{\Gamma\left(\pi^{-} 3 \pi^{0}\right)}{\Gamma_{e}}$ & $0.0157 \pm 0.2 \%$ & $0.01621 \pm 0.2 \%$ & 0.01622 \\
$\frac{\Gamma\left(2 \pi^{-} \pi^{+} \pi^{0}\right)}{\Gamma_{e}}($ no $\omega)$ & $0.0486 \pm 0.2 \%$ & $0.02703 \pm 0.2 \%$ & 0.02706 \\
\hline
\end{tabular}

\footnotetext{
${ }^{5}$ These results differ from [9] by a factor of $(243 / 2 n !) \cos ^{2} \theta_{c}$, where $n$ denotes the number of identical pions in the final state.
} 
We have also checked that, in the narrow $\rho$-width approximation and for massless pions, we reproduce relation (4.14) from ref. [9]. ${ }^{6}$

\begin{tabular}{|l|l|l|l|}
\hline Decay mode & MC result & $\begin{array}{l}\text { MC result } \\
\left(m_{\pi}=0\right)\end{array}$ & $\begin{array}{l}\text { Semi-analytical result } \\
\text { narrow width }\end{array}$ \\
\hline$\frac{\Gamma\left(\pi^{-} \omega\left(\pi^{-} \pi^{+} \pi^{0}\right)\right)^{*}}{\Gamma_{e}}$ & $0.02746 \pm 0.1 \%$ & $0.1818 \pm 0.1 \%$ & $0.17997\left(m_{\pi}=0\right)$ \\
$\frac{\Gamma\left(\pi^{-} \omega\left(\pi^{-} \pi^{+} \pi^{0}\right)\right)}{\Gamma_{e}}$ & $0.03308 \pm 0.15$ & & $0.02715\left(m_{\pi}=0.1395\right)$ \\
\hline
\end{tabular}

* The cross sections from the first line of this table were calculated exceptionally with different input parameters: $\sigma=0, m_{\rho}=0.768, \Gamma_{\rho}=0.149, \Gamma_{\omega}=0.0084$.

We have checked that up to $1 \%(0.2 \%$ if the $\omega$ width is decreased with respect to the physical value) we reproduce the ratio $\Gamma(\pi \pi \gamma) / \Gamma(\pi \omega(3 \pi)=8.5 / 88.8$ (see ref. [3]), consistent with our input for the $\omega$ decay rates.

The rate $\Gamma\left(\pi^{+} \pi^{+} \pi^{-} \pi^{0}\right) / \Gamma_{e}=0.0815 \pm 0.1 \%$ was calculated with the full matrix element and including mass terms as defined in this chapter. This is about a factor of 2 to 4 too small in the case of $\tau \rightarrow \pi \omega \nu$ decay and also about a factor of 4 too small in the case of the non- $\omega$ contribution to the $\pi^{-} \pi^{-} \pi^{+} \pi^{0} \nu$ decay channel of the $\tau$. Possible improvements will be presented in the concluding remarks.

\section{MODIFICATIONS IN THE ALGORITHM}

In the construction of this update we preserve the compatibility with the previously published versions of TAUOLA, including the numerical values of the coupling constants, masses, etc. Since the form factors used in the hadronic currents may change in future versions of the program, as a consequence of improved data, we treat the resonance parameters as locally defined variables, which may even differ from one form-factor to another. Masses of the "resonances" are also defined in the routine CHOICE, which defines the parameters for the phase-space pre-sampling. Note that an inappropriate choice of these parameters may affect the accuracy of the program in case of a substantial percentage of overweighted events.

The content of the common block COMMON /TAUNPI/CBRNPI $(4), \operatorname{AMAS}(6,4), \operatorname{KPI}(6,4)$ MULT (4) remains unchanged. However, the matrix CBRNPI is now a dummy. The meaning of the entries in common blocks TAUBRA and TAUBMC remains the same. Decay modes are ordered as in the abstract of the paper.

\footnotetext{
${ }^{6}$ We assume that in this paper, as a rule, all statistical factors are omitted.
} 
Two new common blocks are introduced. The first one COMMON /TAUPK/ IDKPI $(3,7)$ collects the internal flags of final-state particles for the new three-hadron decay modes. The second one COMMON /TAUKLE/ BRA1, BRKO, BRKOB, BRKS should be initialized with the following numerical input: $0.5,0.5,0.5,0.667$. As an option the values 1 or 0 can be used. Choosing the respective parameters to 1 forces respectively $a_{1}$ decay to three charged pions, $K_{0}\left(\bar{K}_{0}\right)$ to turn always into $K_{S}$, and finally $K^{* \pm}$ to decay into $K^{0} \pi^{ \pm}$; choosing 0 forces respectively $a_{1} \rightarrow 2 \pi^{0} \pi^{ \pm}$decay, $K_{0}\left(\bar{K}_{0}\right)$ to turn always into $K_{L}$, and $K^{* \pm}$ to decay into $K^{ \pm} \pi^{0}$.

The content of the commons TAUBRA and TAUKLE can be redefined before every individual decay of $\tau^{+}$or $\tau^{-}$. It is thus possible, for instance, to force the composition of the decay sample to be different for $\tau^{+}$and for $\tau^{-}$in $\tau$-pair decays.

\section{CONCLUDING REMARKS}

Let us summarize the main features of the Monte Carlo algorithm/program presented so far:

- The calculation, as implemented in the Monte Carlo, is based on the orthodox quantum mechanics - this is especially important for spin effects.

- Phase space is treated independently from the matrix element, and the masses of all final-state particles are included in appropriate Jacobian factors.

- All the physics is located in the matrix element that features intermediate-state resonances as an enhancement in the hadronic current part. In the program the matrix element is located and isolated in a well-defined program module.

Tests consisting of comparison of numerical results obtained from the program and independent semi-analytical calculations are described and listed here or in refs. [1, 7].

Let us recall standard requirements [23], which one would like to have for the tool to be used in data analysis. It is assumed that a certain contribution to the systematic error can be neglected if it is a factor of three smaller than the anticipated precision of the measurement to be performed. This requirement can be easily fulfilled in case of Monte Carlo for QED defined processes, where calculations can be performed with arbitrary precision (at least in principle) and question of physics systematic error can be addressed in the framework of the calculations performed at different perturbation orders, see ref. [24] as an example.

In the case of $\tau$ decays the hadronic current $J^{\mu}$ has to be related (fitted) to experimental data. That influences significantly the status of the simulation program and the related "theoretical" error. In particular, the parametrization of the form-factors includes the statistical error of the data used in its definition. In the best case (contrary to QED) the physical precision of the resulting tool is thus larger by a factor of 3 ( $3 \sigma$ standard) than this error and thus larger by a factor of 9 , at best, than what one one would like. 
Otherwise unwanted correlation between different experiments may be introduced in this way. Of course the above extreme scenario takes place when the hadronic current in the Monte Carlo is parametrized, only and entirely, with the help of the another external experiment. In practice, any experiment with new, better data will fit the hadronic current with its own data. Nevertheless the above remark should be kept in mind.

An advanced user of TAUOLA may be interested in trying other possible choices of the hadronic-current parametrization, such as the ones defined in [21, 17] or any other. This is rather straightforward and only requires replacement of well-defined routines defining the hadronic current.

Good candidates for such improvements are the $2 \pi^{-} \pi^{+} \pi^{0}, \pi^{-} 3 \pi^{0}$ and $\pi^{-} \pi^{0} \gamma$ decay modes, where our ansatz seems to be in disagreement with the experimental results [22]. For the $\omega$-enhanced currents it can be argued that the coupling constant $g_{\rho \omega \pi}$ is not necessarily the same for the initial-state $\rho$ with off the mass shell as for the final state $\rho \equiv \gamma$ with zero mass. Also, the function $F\left(q^{2}\right)$ used in formulae (28) and (34) could be replaced by $B_{\rho}\left(q^{2}\right)$ or $B_{\rho}^{1}\left(q^{2}\right)$. Both these changes can be used to increase an overall $\tau$-decay branching ratio into $\pi \omega$. The situation in case of a chiral Lagrangian current is more complicated. Replacement of $B_{\rho}\left(q^{2}\right)$ in formulae (31) and (33) by $B_{\rho}^{1}\left(q^{2}\right)$ can be a straightforward partial solution. However, incorporation of the enhancements due to $\rho^{-}$, which were experimentally detected in $\tau^{-}$decays is difficult in our ansatz, because it breaks current conservation [9]. We think that the form of the current directly chosen to reproduce data without theoretical constraints should be used in this case. We prefer to stay for a while with our solution suitable for tests of technical precision of the Monte Carlo, leaving the choice to the users of the program.

\section{Acknowledgements}

This work was supported in part by the Polish Government grants KBN-203809101 and KBN-223729102 and by German grant BMFT 05-5KA 94P. We would like to thank E. Mirkes for numerous discussions and for providing us with numerical results prior to publication. Useful discussion with A. M. Lutz of the ALEPH collaboration, A. Weinstein of CLEO II, M. Gruenewald of L3, K. Strahl and R. Waldi of ARGUS, and M. Jeżabek are also acknowledged. One of the authors (ZW) acknowledges partial support of the Heraeus Stiftung, Germany.

\section{References}

[1] S. Jadach, J. H. Kühn and Z. Wạs, Comp. Phys. Comm. 64 (1991) 275.

[2] S. Jadach and Z. Wạs, "QED predictions and their systematic errors", CERN preprint CERN TH-6195 and proceedings of the "MC91" conference, ed. B. van Eijk, Amsterdam 1991.

[3] M. Aguilar-Benitez et al. (Particle Data Group), 'Review of Particle Properties', Phys. Rev. D45 (1992) vol. 11 
[4] S. Jadach and Z. Wa̧s, Comp. Phys. Commun. 36 (1985) 191, ibid. 64 (1991) 275 .

[5] S. Jadach, B. F. L. Ward and Z. Wạs, Comp. Phys. Comm. 66 (1991) 276.

[6] E. Barberio, B. van Eijk and Z. Wạs, Comp. Phys. Commun. 66 (1991) 115.

[7] S. Jadach, M. Jeżabek, J. H. Kühn and Z. Wạs, Comp. Phys. Comm. 70 (1992) 69.

[8] S. Jadach, Acta Phys. Polon. B16 (1985) 1007.

[9] R. Fischer, J. Wess and F. Wagner, Z. Phys. C3 (1980) 313.

[10] J.H. Kühn and F. Wagner, Nucl. Phys. B236 (1984) 16.

[11] J.H. Kühn and X. Santamaria, Z. Phys. C48 (1990) 445.

[12] R. Decker, E. Mirkes, R. Sauer and Z. Wa̧s, Karlsruhe preprint TTP92/25 (1992) Z. Phys. in press.

[13] H. Georgi, Weak interactions and modern particle theory (The Benjamin/Cummings Pub. Co., Inc., 1984).

[14] N. Isgur, C. Morningstar and C. Reader, Phys. Rev. D39 (1989) 1357.

[15] G. Kramer, W. F. Palmer and S. S. Pinsky, Phys. Rev. D30 (1984) 89.

[16] G. Kramer and W. F. Palmer, Z. Phys. C 25 (1984) 195.

[17] J. J. Gomez-Cadenas, M. C. Gonzales-Garcia and A. Pich, Phys. Rev. D42 (1990) 3093.

[18] E. Mirkes and J. H. Kühn, Phys. Lett. B286 (1992) 381, and TTP92/20, Z. Phys. C56 (1992) 661.

[19] E. Mirkes, private communication.

[20] R. Decker, Z. Phys. C36 (1987) 487.

[21] M. Feindt, Z. Phys. C48 (1990) 681.

[22] H. Albrecht et al., Phys. Lett. B260 (1991) 259.

[23] F. Dydak et al., "Electroweak radiative corrections at LEP energies", CERN 87-08 (1987), vol. 1, p. 157.

[24] S. Jadach, E. Richter-Wạs, B. F. L. Ward and Z. Wạs, Phys. Lett. B268 (1991) 253. 
DEMOIISTRATIOII PROGRAM

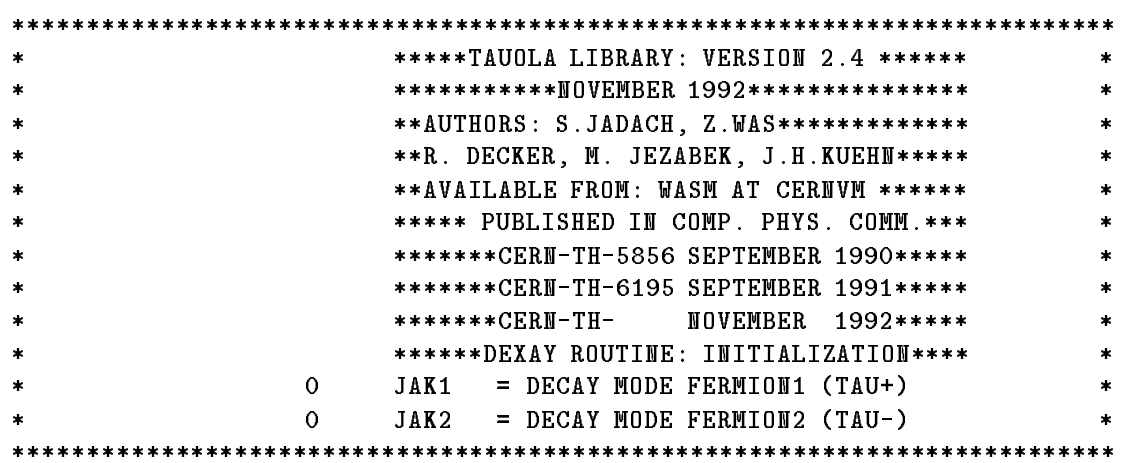

\begin{tabular}{|c|c|c|c|c|c|c|c|c|c|c|c|}
\hline \multirow[b]{2}{*}{ I } & \multirow[b]{2}{*}{ particle } & \multicolumn{5}{|c|}{ Event listing (standard) } & \multirow[b]{2}{*}{$P(I, 1)$} & \multirow[b]{2}{*}{$P(I, 2)$} & \multirow[b]{2}{*}{$P(I, 3)$} & \multirow[b]{2}{*}{$P(I, 4)$} & \multirow[b]{2}{*}{$P(I, 5)$} \\
\hline & & $K(I, 1)$ & $K(I, 2)$ & $K(I, 3)$ & $K(I, 4)$ & $K(I, 5)$ & & & & & \\
\hline 1 & $! e+!$ & 21 & -11 & 0 & 3 & 4 & .00000 & .00000 & 1.78420 & 1.78420 & .00051 \\
\hline 2 & $! e^{-!}$ & 21 & 11 & 0 & 3 & 4 & .00000 & .00000 & -1.78420 & 1.78420 & .00051 \\
\hline 3 & tau+ & 1 & -15 & 1 & 0 & 0 & .00000 & .00000 & .00000 & 1.78420 & 1.78420 \\
\hline 5 & nu_tau & 1 & 16 & 4 & 0 & 0 & .15368 & .30209 & .02964 & .34037 & .00998 \\
\hline 6 & $(W-)$ & 11 & -24 & 4 & 7 & 9 & -.15368 & -.30209 & -.02964 & 1.44382 & 1.40316 \\
\hline 7 & eta & 1 & 221 & 6 & 0 & 0 & -.05243 & -.18715 & -.30979 & .65949 & .54880 \\
\hline 8 & pi- & 1 & -211 & 6 & 0 & 0 & -.25587 & -.31094 & .26133 & .49992 & .13957 \\
\hline \multirow[t]{2}{*}{9} & pio & 1 & 111 & 6 & 0 & 0 & .15461 & .19600 & .01882 & .28441 & .13496 \\
\hline & & Im charg & .00 & sum $m$ & omentum and & inv. mass & .00000 & .00000 & .00000 & 3.56839 & 3.56839 \\
\hline
\end{tabular}

\begin{tabular}{|c|c|c|c|}
\hline * & & $* * * * * * * *$ DADMEL FIIIAL REPORT & * \\
\hline * & 864418 & HEVRAW = HO. OF EL DECAYS TOTAL & * \\
\hline * & 210462 & NEVACC = NO. OF EL & * \\
\hline * & 0 & IEVOVR = IOO. OF OVERWEIGHTED EVENTS & * \\
\hline * & $.41100 \mathrm{E}-12$ & PARTIAL WTDTH ( ELECTRON) IN GEV UNITS & * \\
\hline * & .994740903 & III UIITS GFERMI**2*MASS**5/192/PI**3 & * \\
\hline * & .001008425 & RELATIVE ERROR OF PARTIAL WIDTH & * \\
\hline * & & COMPLETE QED CORRECTIONS INCLUDED & * \\
\hline * & & BUT ONLY V-A CUPLINGS & * \\
\hline
\end{tabular}

\begin{tabular}{|c|c|c|c|}
\hline * & & ********* DADMIUU FIIIAL REPORT $* * * * * * * * *$ & $*$ \\
\hline * & 892266 & IEVRAW = NO. OF MU DECAYS TOTAL & * \\
\hline * & 211459 & NEVACC $=\mathbb{N O}$. OF MU DECS. ACCEPTED & * \\
\hline * & 0 & IEVOVR = IO. OF OVERWEIGHTED EVENTS & * \\
\hline * & $.40046 \mathrm{E}-12$ & PARTIAL WTDTH (MU DECAY) III GEV UHITS & * \\
\hline * & .969211578 & III UIITS GFERMI**2*MASS**5/192/PI**3 & * \\
\hline * & .001089378 & RELATIVE ERROR OF PARTIAL WIDTH & * \\
\hline * & & COMPLETE QED CORRECTIOHS IIICLUDED & * \\
\hline * & & BUT ONLY V-A CUPLIIIGS & * \\
\hline \multicolumn{4}{|c|}{ 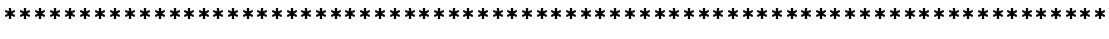 } \\
\hline \multicolumn{4}{|c|}{ 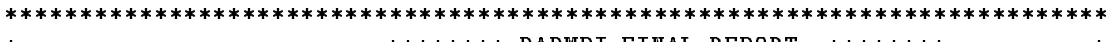 } \\
\hline * & & $* * * * * * * *$ DADMPI FIIIAL REPORT $* * * * * * * *$ & * \\
\hline * & 210704 & HEVTOT = HO. OF PI DECAYS TOTAL & * \\
\hline * & $.25031 \mathrm{E}-12$ & PARTIAL WTDTH ( PI DECAY) III GEV UHITS & * \\
\hline * & .605813980 & III UNITS GFERMI**2*MASS**5/192/PI**3 & * \\
\hline * & .00000000 & RELATIVE ERROR OF PARTIAL WIDTH (STAT.) & * \\
\hline
\end{tabular}




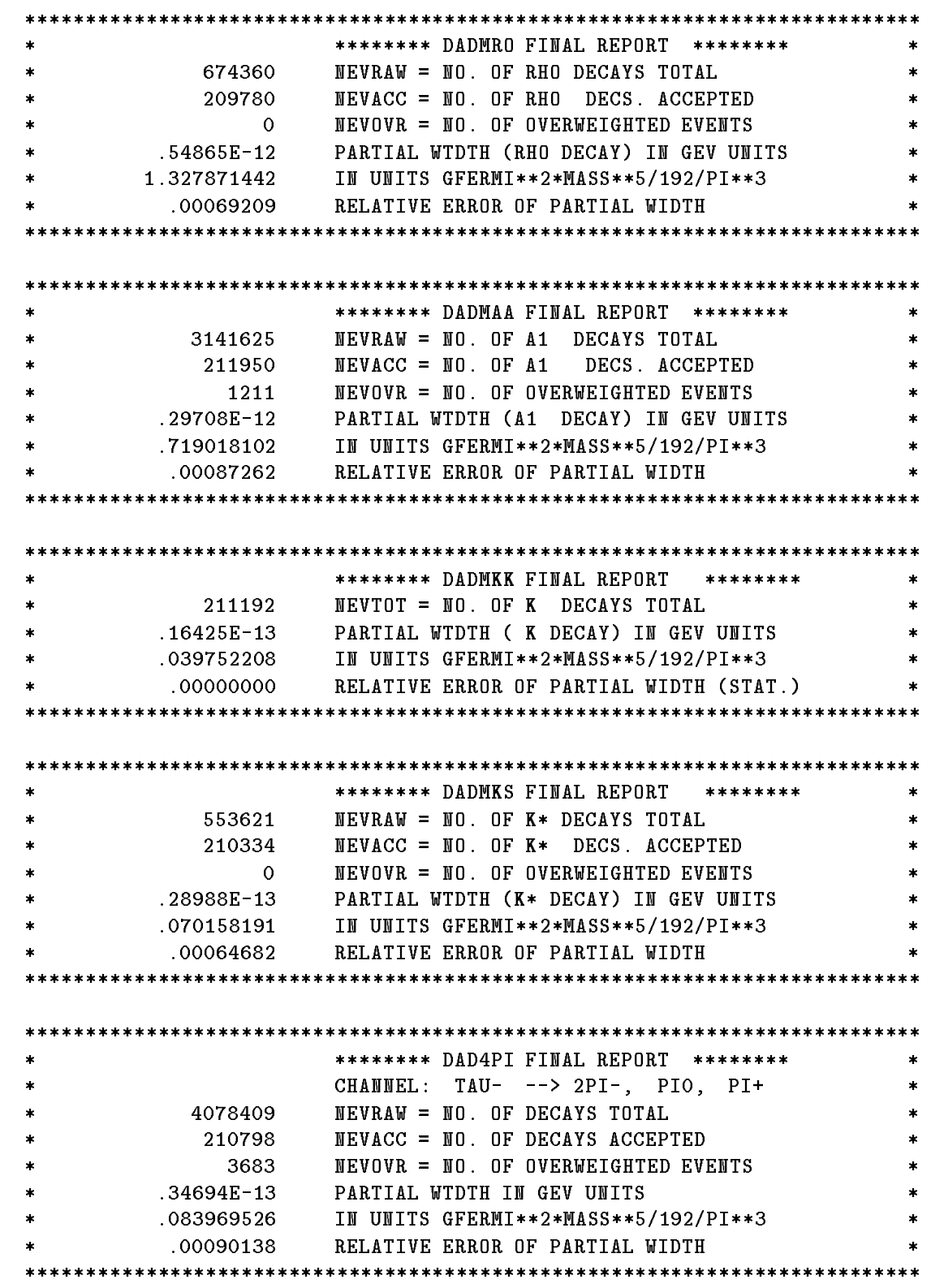




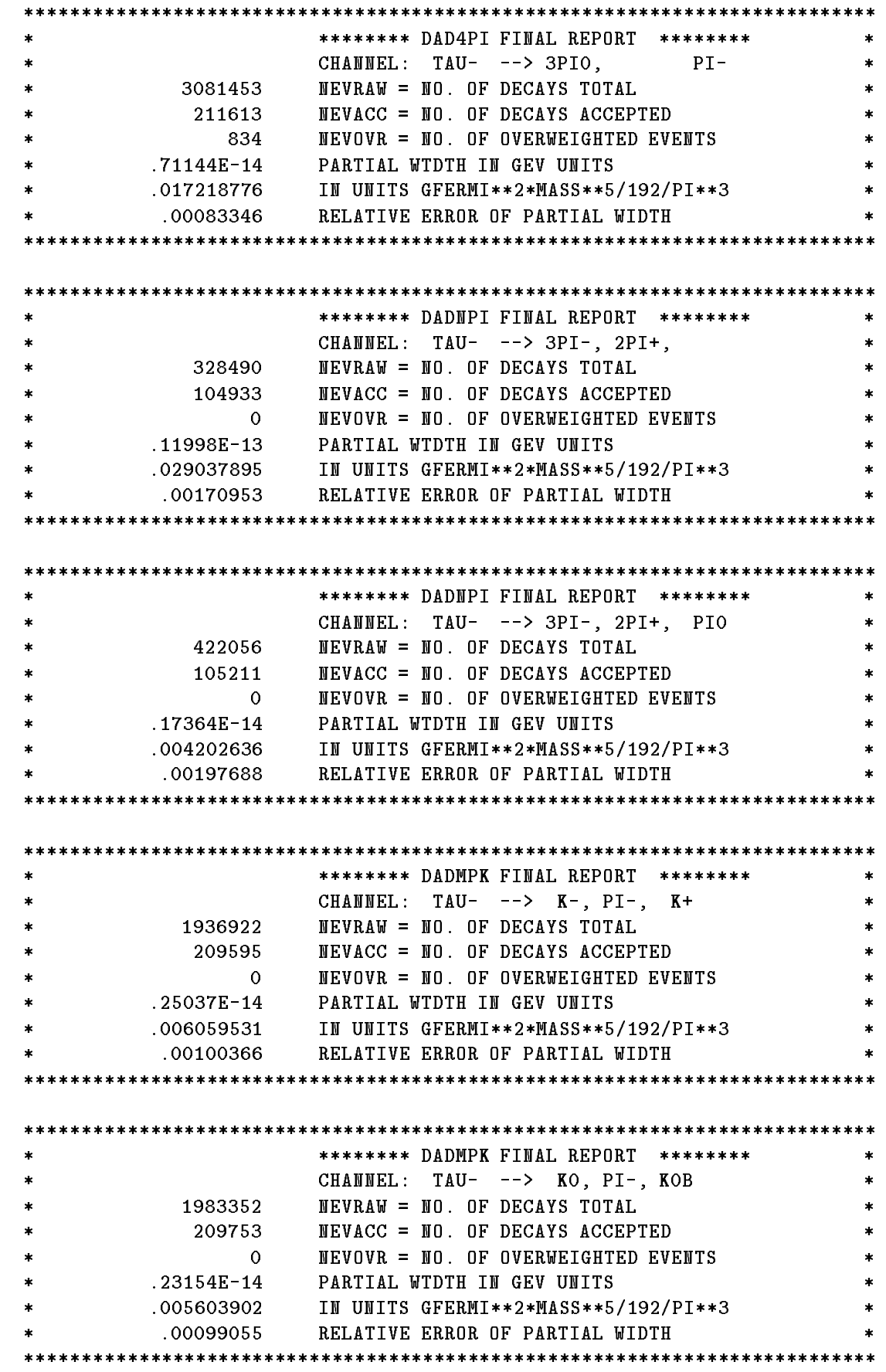




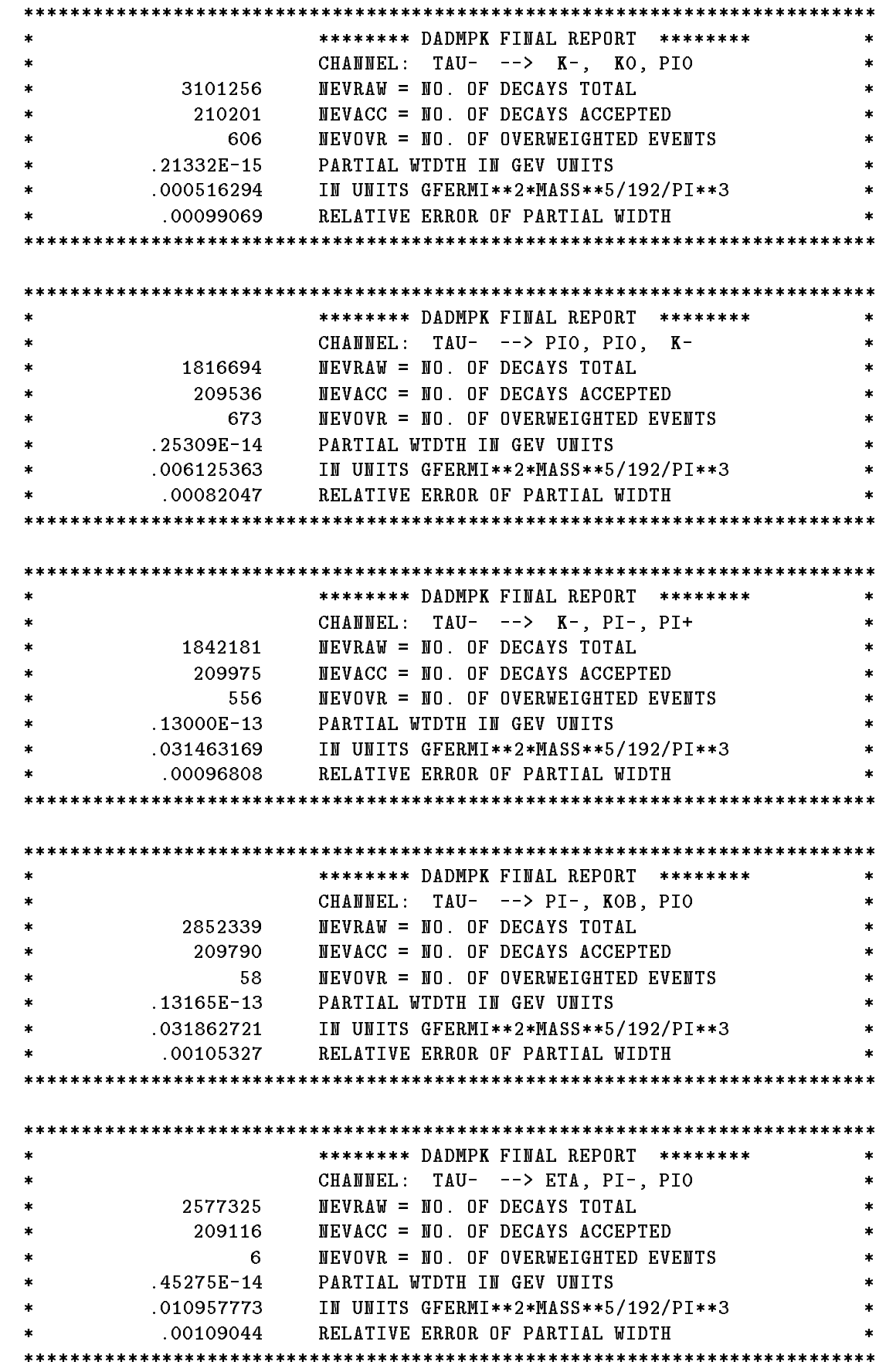




\begin{tabular}{|c|c|c|c|c|c|}
\hline \multicolumn{3}{|c|}{ * } & \multicolumn{3}{|c|}{$* * * * * * * *$ DADMPK FIIIAL REPORT $* * * * * * * *$} \\
\hline \multicolumn{3}{|c|}{ * } & \multicolumn{3}{|c|}{ CHANNEL: TAU- $\quad->$ PI-, PIO, GAM } \\
\hline \multicolumn{3}{|r|}{1046191} & \multicolumn{2}{|c|}{ NEVRAW = $\mathbb{H} O$. OF DECAYS TOTAL } & * \\
\hline \multicolumn{2}{|l|}{ * } & 211190 & \multicolumn{2}{|c|}{ NEVACC $=$ IIO. OF DECAYS ACCEPTED } & * \\
\hline \multicolumn{2}{|l|}{ * } & 47 & \multicolumn{2}{|c|}{ HEVOVR = HO. OF OVERWEIGHTED EVEHTS } & * \\
\hline \multicolumn{2}{|l|}{ * } & $13106 \mathrm{E}-14$ & \multicolumn{2}{|c|}{ PARTIAL WTDTH III GEV UNITS } & * \\
\hline \multicolumn{2}{|l|}{ * } & .003172031 & \multicolumn{2}{|c|}{ III UNITS GFERMI**2*MASS $* * 5 / 192 / \mathrm{PI} * * 3$} & * \\
\hline \multicolumn{2}{|c|}{ * } & .00085179 & \multicolumn{2}{|c|}{ RELATIVE ERROR OF PARTIAL WIDTH } & * \\
\hline \multicolumn{6}{|c|}{ 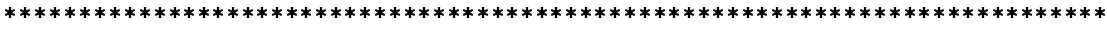 } \\
\hline \multicolumn{6}{|c|}{ 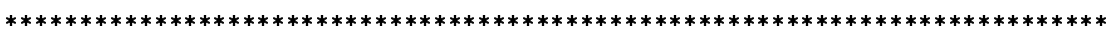 } \\
\hline \multicolumn{3}{|c|}{ * } & \multicolumn{2}{|c|}{$* * * * *$ TAUOLA LIBRARY : VERSIOII $2.4 * * * * * *$} & * \\
\hline \multicolumn{3}{|l|}{ * } & \multicolumn{2}{|c|}{$* * * * * * * * * * * \mathbb{I I O V E M B E R ~} 1992 * * * * * * * * * * * * * * *$} & * \\
\hline \multicolumn{3}{|l|}{ * } & \multicolumn{2}{|c|}{ **AUTHORS : S.JADACH, Z.WAS************* } & * \\
\hline * & & & $* * R$. DECKER, M. JEZABE & K , J.H.KUEHII***** & * \\
\hline * & & & **AVAILABLE FROM: WASM & I AT CERIVI $* * * * * *$ & * \\
\hline * & & & ***** PUBLISHED III COM & IP. PHYS. COMM .*** & * \\
\hline * & & & $* * * * * * * \mathrm{CERNI}-\mathrm{TH}-5856 \mathrm{SE}$ & PTEMBER $1990 * * * * *$ & * \\
\hline * & & & $* * * * * * * \mathrm{CERN}-\mathrm{TH}-6195 \mathrm{SE}$ & PTEMBER $1991 * * * * *$ & * \\
\hline * & & & $* * * * * * * \mathrm{CERNI}-\mathrm{TH}-\quad$ IIO & JVEMBER $1992 * * * * *$ & * \\
\hline * & & & ******DEXAY ROUTINE: F & IIIAL REPORT****** & * \\
\hline * & & 0 & $\mathbb{N E V 1}=\mathbb{H O}$. OF TAU+ D & ECS . ACCEPTED & * \\
\hline * & & 2000000 & $\mathbb{N E V} 2=\mathbb{N O}$. OF TAU- D & ECS . ACCEPTED & * \\
\hline * & & 2000000 & HEVTOT = SUM & & * \\
\hline * & NOEVTS & PART . WIDTH & ROUT INE & DECAY MODE & * \\
\hline * & 105253 & .9947409 & .0010084 & ELECTROII & * \\
\hline * & 105545 & .9692116 & .0010894 & MUOW & * \\
\hline * & 105285 & .6058140 & .0000000 & PIOII & * \\
\hline * & 104835 & 1.3278714 & .0006921 & $\mathrm{RHO}(->2 \mathrm{PI})$ & * \\
\hline * & 105955 & .7190181 & .0008726 & A1 $\quad(->3 P I)$ & * \\
\hline * & 105616 & .0397522 & .0000000 & KAOII & * \\
\hline * & 105047 & .0701582 & .0006468 & $\mathrm{~K} *$ & * \\
\hline * & 105605 & .0839695 & .0009014 & $\mathrm{PI}-2 \mathrm{PI}+\mathrm{PIO}$ & * \\
\hline * & 105846 & .0172188 & .0008335 & PI- 3PI0 & * \\
\hline * & 104933 & .0290379 & .0017095 & $3 \mathrm{PI}-2 \mathrm{PI}+$ & * \\
\hline * & 105211 & .0042026 & .0019769 & $3 P I-2 P I+P I 0$ & * \\
\hline * & 104818 & .0060595 & .0010037 & $\mathrm{~K}+\mathrm{K}-\mathrm{PI}+$ & * \\
\hline * & 105154 & .0056039 & .0009906 & KOB KO PI+ & * \\
\hline * & 105108 & .0005163 & .0009907 & $\mathrm{~K}+\mathrm{KOB}$ PIO & * \\
\hline * & 105138 & .0061254 & .0008205 & K+ PIO PIO & * \\
\hline * & 105070 & .0314632 & .0009681 & $\mathrm{~K}+\mathrm{PI}-\mathrm{PI}+$ & * \\
\hline * & 105347 & .0318627 & .0010533 & KO PIO PI+ & * \\
\hline * & 104598 & .0109578 & .0010904 & ET PI- PIO & * \\
\hline * & 105636 & .0031720 & .0008518 & PI-PIO GAM & * \\
\hline * & & THE & ERROR IS RELATIVE AID & PART . WIDTH & * \\
\hline * & & III & IITS GFERMI**2*MASS**5/ & $192 / \mathrm{PI} * * 3$ & * \\
\hline
\end{tabular}

